\title{
Modeling the impact of delaying transcatheter aortic valve replacement for the treatment of aortic stenosis in the era of COVID-19
}

Daniel R. Freno, MD, ${ }^{a}$ Maren E. Shipe, MD, MPH, ${ }^{\mathrm{b}}$ Melissa M. Levack, MD, ${ }^{\mathrm{c}}$ Ashish S. Shah, MD, Stephen A. Deppen, PhD, ${ }^{\text {a,d }}$ Jared M. O'Leary, MD, ${ }^{e}$ and Eric L. Grogan, MD, MPH, FACS ${ }^{a, d}$

\section{ABSTRACT}

Objective: The aim of this study was to model the short term and 2-year overall survival (OS) for intermediate-risk and low-risk patients with severe symptomatic aortic stenosis (AS) undergoing timely or delayed transcatheter aortic valve replacement (TAVR) during the 2019 novel coronavirus (COVID-19) pandemic.

Methods: We developed a decision analysis model to evaluate 2 treatment strategies for both low-risk and intermediate-risk patients with AS during the COVID-19 novel coronavirus pandemic.

Results: Prompt TAVR resulted in improved 2-year OS compared with delayed intervention for intermediate-risk patients (0.81 vs 0.67 ) and low-risk patients (0.95 vs 0.85), owing to the risk of death or the need for urgent/emergent TAVR in the waiting period. However, if the probability of acquiring COVID-19 novel coronavirus is $>55 \%$ (intermediate-risk patients) or $47 \%$ (low-risk patients), delayed TAVR is favored over prompt intervention ( 0.66 vs 0.67 for intermediate risk; 0.84 vs 0.85 for low risk).

Conclusions: Prompt transcatheter aortic valve replacement for both intermediate-risk and low-risk patients with symptomatic severe AS results in improved 2-year survival when local healthcare system resources are not significantly constrained by the COVID-19. (JTCVS Open 2021;7:63-71)

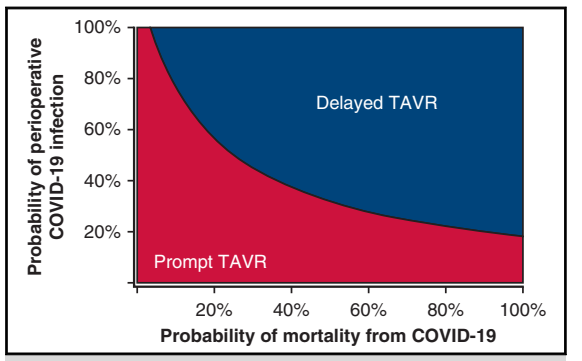

Results from decision analysis favor prompt TAVR over delayed TAVR.

\section{CENTRAL MESSAGE}

TAVR for both intermediate-risk and low-risk patients with symptomatic severe AS should not be delayed during the COVID-19 pandemic unless local hospital resources are significantly constrained.

\section{PERSPECTIVE}

As the 2019 novel coronavirus (COVID-19) began to spread around the world, many healthcare systems ceased elective procedures in anticipation of a surge in COVID-19 patients and in an effort to protect patients undergoing elective procedures. Guidance is needed to help clinicians and healthcare leaders determine whether TAVR should be delayed for the treatment of symptomatic AS during the COVID-19 pandemic.

See Commentaries on pages 72 and 74

\footnotetext{
From the Departments of ${ }^{\mathrm{a} T h o r a c i c ~ S u r g e r y, ~}{ }^{\mathrm{b}}$ General Surgery, ${ }^{\mathrm{c}}$ Cardiac Surgery, and ${ }^{\mathrm{e}}$ Cardiovascular Medicine, Vanderbilt University Medical Center, Nashville, Tenn; and ${ }^{\mathrm{d}}$ Tennessee Valley Healthcare System, Department of Surgery, Nashville, Tenn.

Drs Freno and Shipe contributed equally and should be considered co-first authors. Dr Shipe is supported by the Agency for Healthcare Research (Grant T32 HS026122). Dr Grogan's research time is supported by the Department of Veterans Affairs. The views expressed in this article are those of the authors and do not necessarily represent the views of the Department of Veterans Affairs. Dr Deppen received support from the Early Detection Research Network (NCI-5U24-CA0866368). The funding agency had no role in the design and conduct of the study; collection, manage-
}

ment, analysis, and interpretation of the data; preparation, review, or approval of the manuscript; or the decision to submit the manuscript for publication.

Received for publication Aug 4, 2020; accepted for publication June 2, 2021; available ahead of print July 28, 2021.

Address for reprints: Eric L. Grogan, MD, MPH, FACS, Department of Thoracic Surgery, 609 Oxford House, 1313 21st Ave South, Nashville, TN 37232 (E-mail: eric. grogan@vumc.org).

2666-2736

Copyright $(C) 2021$ The Authors. Published by Elsevier Inc. on behalf of The American Association for Thoracic Surgery. This is an open access article under the CC BY-NCND license (http://creativecommons.org/licenses/by-nc-nd/4.0/).

https://doi.org/10.1016/j.xjon.2021.06.006 


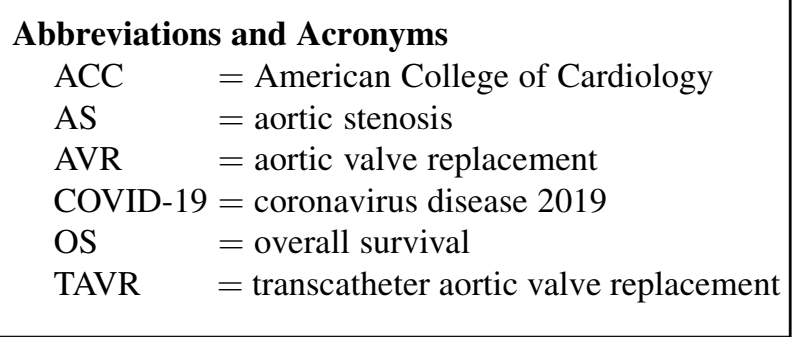

Coronavirus disease 2019 (COVID-19) began spreading around the globe in early 2020 and was designated a global pandemic on March 11, 2020. ${ }^{1}$ Healthcare systems worldwide have been dramatically impacted as they have been forced to refocus their efforts on combating COVID-19. The world was watching as reports from Lombardy, Italy described hospitals at capacity and short on resources. ${ }^{2}$ In anticipation of a similar surge in cases and stress on US healthcare systems, on March 13, 2020, the American College of Surgeons released guidelines for the management of elective surgical procedures encouraging providers and hospitals to reschedule all elective surgical procedures. ${ }^{3}$ Shortly thereafter, the US Surgeon General and the Centers for Medicare and Medicaid Services also released guidance stipulating that all elective surgeries be delayed during the pandemic. ${ }^{4}$ These measures were intended both to protect patients undergoing elective procedures and to safeguard the healthcare system, preserving the capacity to deal with a surge in COVID-19 patients.

Professional societies, such as the American College of Cardiology (ACC), have recognized the impact that these guidelines have had on the ability to care for patients with symptomatic aortic valve stenosis (AS), putting these patients at increased risk for cardiovascular complications and death. Once symptoms develop as a result of AS, 2year mortality approaches $50 \%$ if not treated with aortic valve replacement. ${ }^{5,6}$ The ACC and the Society for Cardiovascular Angiography and Interventions released a consensus statement on triage of structural heart disease patients and timing of intervention. For patients with severe symptomatic AS and reduced ejection fraction considered secondary to AS, the presence of class III-IV congestive heart failure, or syncope, transcatheter aortic valve replacement (TAVR) should be considered on an inpatient basis. However, for patients who are minimally symptomatic without any of the previously stated characteristics, the recommendation was for either immediate TAVR or delayed TAVR with close clinical follow-up. ${ }^{7}$

The risk of delaying the treatment of symptomatic AS must be balanced with the risk of mortality from COVID-19 infection. Early reports suggest that hospitalized patients, especially those with significant comorbidities, such as cardiovascular and respiratory disease, are at increased risk for complications, including death from COVID-19, whether community or hospital-acquired..$^{8-11}$ In addition, it has been well established that patients age $>60$ years are at increased risk for complications and COVID-related mortality. ${ }^{11-15}$

The purpose of this study was to compare the 2-year outcomes of delaying transfemoral TAVR for 6 months in lowand intermediate-risk patients with severe symptomatic AS with possible perioperative COVID-19 infection arising in the immediate postoperative period. This model seeks to provide clinicians and policy leaders with guidance on the safety of prompt TAVR during an active phase of community spread of COVID-19 versus delaying the procedure until community spread has decreased dramatically.

\section{METHODS \\ Decision Model Design}

We developed a decision analysis model to evaluate 2 treatment strategies for AS during the COVID-19 pandemic: prompt transfemoral TAVR and delayed transfemoral TAVR after 6 months (Figure 1). The decision tree details the initial choice (ie, the decision node) of proceeding promptly with TAVR or delaying TAVR and follows branch points to the ultimate outcomes of death or 2-year overall survival (OS) (terminal nodes). The availability of hospital resources can be a factor in the initial choice of delaying TAVR if local levels of COVID-19 infection are sufficiently high such that hospital beds and ventilators are unavailable. If the operation is performed electively for either prompt or delayed TAVR, there are chance nodes for operative mortality, perioperative COVID-19 infection, and mortality due to COVID-19. For the delayed pathway, there is a chance that the patient will not undergo elective TAVR but may decompensate and require urgent or emergent TAVR either from disease progression during the interim or from contracting COVID-19 (see below). For a patient undergoing urgent or emergent TAVR, there are again chance nodes for operative mortality, perioperative COVID-19 infection, and mortality due to COVID-19. Perioperative COVID-19 infection is defined as having contracted the virus in during a period of several days before or during the hospitalization for the procedure.

We used TreeAge Pro version 2018 (TreeAge Software, Williamson, Mass) to construct the decision tree model. A literature review and expert opinion (when published data were unavailable) were used to estimate model parameters and ranges for sensitivity analysis. Individual patient data were not used for this study, and thus informed consent was not required. Institutional Review Board approval was not necessary, because this study did not involve biomedical research.

\section{Patient Simulations}

We modeled 2 clinical scenarios: intermediate risk and low risk, as defined by the patient's STS risk for isolated surgical AVR. Whereas our intervention is TAVR, current methodology to risk-stratify patients for TAVR in clinical practice and the available literature with regard to TAVR outcomes use the STS risk of mortality for surgical AVR. Lowrisk and intermediate-risk patients were chosen because they pose the most significant challenge when determining the safety of delaying TAVR and because they encompass approximately two-thirds to threequarters of all TAVR procedures in the modern era. ${ }^{16}$ Our intermediaterisk base clinical case was a 75-year-old female presenting with dyspnea and found to have severe AS who was deemed an intermediate-risk candidate for surgical AVR (Table 1). Our low-risk base case was a 65 -year-old female with symptomatic severe AS who was deemed a low-risk candidate for surgical AVR. 


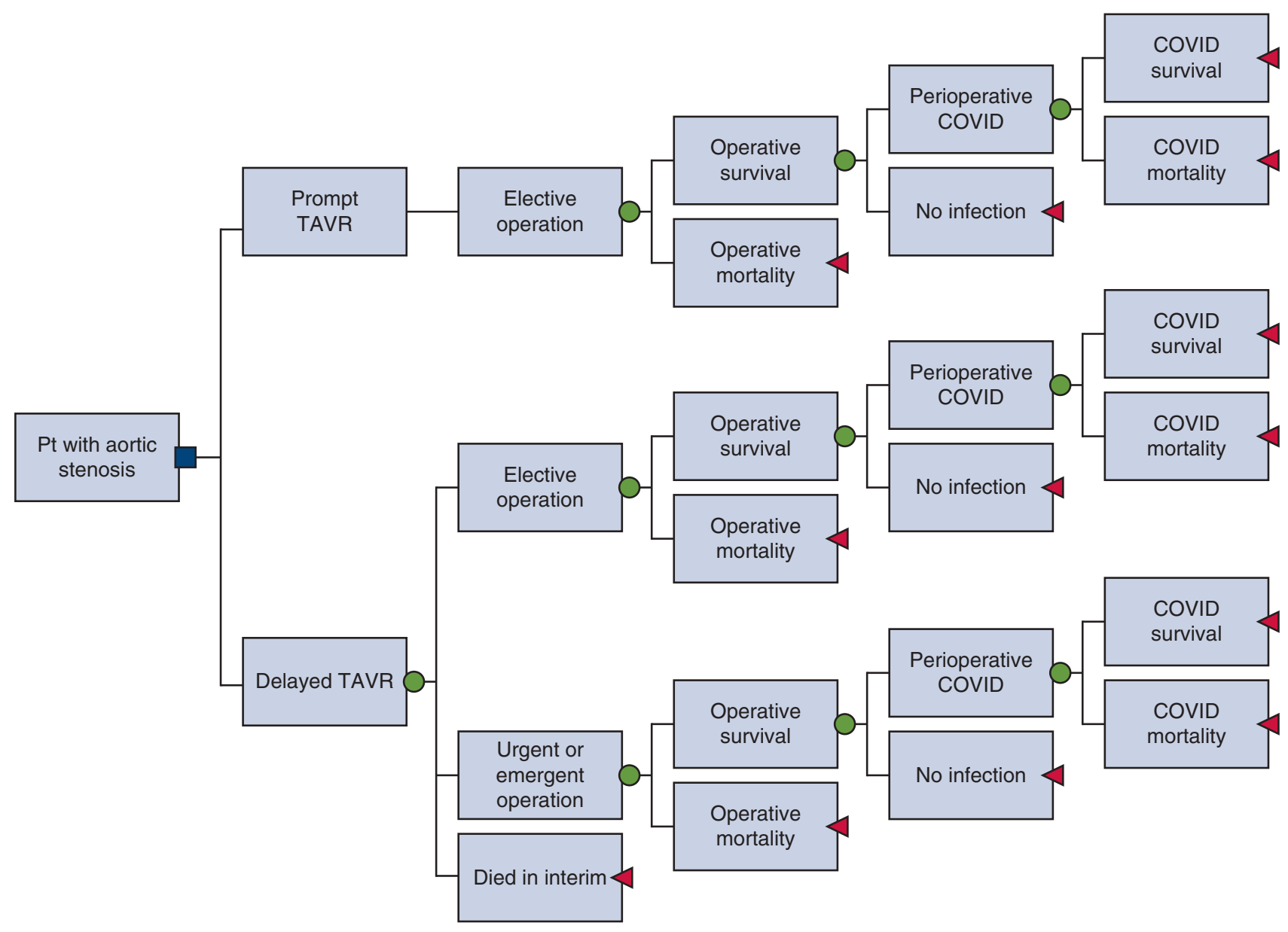

FIGURE 1. Decision analysis tree for the timing of transcatheter aortic valve replacement (TAVR) during the coronavirus disease 2019 (COVID-19) pandemic. The decision analysis tree demonstrates possible outcomes associated with either delaying TAVR or undergoing prompt TAVR. The availability of hospital resources during surges in COVID-19 infections may play a role in the decision to delay TAVR. The blue square indicates the decision node, whether to choose prompt or delayed TAVR; green circles, chance nodes; red triangles, terminal nodes. Numerical values for the chance nodes and terminal nodes are detailed in Tables 2 and 3.

\section{TABLE 1. Clinical scenarios}

\begin{tabular}{|c|c|c|c|c|}
\hline Risk classification & Age, $y$ & Clinical characteristics & STS score, $\%$ & $\begin{array}{c}\text { ACC TAVR in-hospital } \\
\text { mortality risk, } \%\end{array}$ \\
\hline Intermediate-risk patient & 75 & $\begin{array}{l}\text { - Symptomatic AS (dyspnea) } \\
\text { - Mild CAD s/p remote PCI } \\
\text { - Diabetes } \\
\text { - Paroxysmal atrial fibrillation } \\
\text { - } \mathrm{EF}>50 \% \\
\text { - Mild MR } \\
\text { - Creatinine } 1.5 \mathrm{mg} / \mathrm{dL} \\
\text { - Takes clopidogrel }\end{array}$ & 4.5 & 4 \\
\hline Low-risk patient & 65 & $\begin{array}{l}\text { - Symptomatic AS } \\
\text { - Hypertension } \\
\text { - Trivial MR } \\
\text { - } \mathrm{EF}>50 \% \\
\text { - Creatinine } 0.8 \mathrm{mg} / \mathrm{dL}\end{array}$ & 1.3 & 1.5 \\
\hline
\end{tabular}

STS, Society of Thoracic Surgeons; $A C C$, American College of Cardiology; TAVR, transcatheter aortic valve replacement; $A S$, aortic stenosis; $C A D$, coronary artery disease; $P C I$, percutaneous coronary intervention; $E F$, ejection fraction; $M R$, mitral valve regurgitation. 
Both patients were candidates for TAVR via a transfemoral approach and presented to a hospital during the acute phase of the COVID-19 pandemic. This assumes the presence of COVID-19 in the community and hospital, but not to such a degree that hospital resources are exhausted. The institution still has hospital room capacity, available ventilators, and a readily available supply of blood products, and the COVID trajectory is rapidly increasing within the community, necessitating reallocation of resources by the institution. The model assumes that the patients had been screened before the procedure and did not endorse symptoms related to the virus or test negative for COVID-19.

\section{Model Variables}

TAVR. Treatment simulations were performed for both prompt and delayed TAVR. Assumptions for a patient who underwent prompt TAVR were a 2- to 3-day hospitalization, ${ }^{17}$ during which time the patient would be at risk for a hospital-acquired COVID-19 infection, and periprocedural mortality of $1.5 \%$ (low risk) or $4 \%$ (intermediate risk) based on the baseline characteristics (Table 2). ${ }^{27}$

During the 6-month delay in TAVR, there are three possible scenarios: (1) the patient's AS worsened significantly during the interval, necessitating urgent/emergent TAVR; (2) the patient died before undergoing TAVR; or (3) the patient survived without disease progression and underwent electively scheduled delayed TAVR (Table 2). ${ }^{5,6,23,25,26}$ If the patient underwent urgent or emergent TAVR, then the probability of operative mortality increased to $2.3 \%$ (low risk) and $6.1 \%$ (intermediate risk). ${ }^{18-20,27,28}$

COVID-19. The COVID-19 parameters listed in Table 2 were derived from the limited published reports available as of April 15, 2020. An additional variable, risk of perioperative COVID-19 infection, encompasses the risk to patients of both community-acquired and hospital-acquired infection around TAVR. The risk of perioperative COVID-19 infection for prompt TAVR was set at $1.4 \%$ by the research team to reflect the local prevalence of disease as defined by the percentage of positive tests obtained in asymptomatic or minimally symptomatic cases. For delayed TAVR, the risk of perioperative COVID-19 exposure was set to almost zero as the purpose of delaying TAVR for 6 months was to allow the prevalence to decrease. Sensitivity analyses also modeled the risk of perioperative COVID-19 at $0.7 \%$ in the delayed approach in the event that the community prevalence did not drop to zero.
The risk of COVID-19-related mortality after TAVR was derived from reports of patients undergoing major surgery who were subsequently diagnosed with COVID-19 in the postoperative period and is estimated at $21 \%{ }^{21,22}$ These patients were more likely to require intensive care unit (ICU) admission and had an elevated mortality compared with the general non-postoperative patient population age 60 to 79 years. ${ }^{2,15}$

\section{Two-Year OS}

The 2-year OS for TAVR patients similar to our case scenarios was established from the literature (Table 3). ${ }^{29,32}$ Patients who underwent elective TAVR had improved OS compared with those who underwent urgent or emergent TAVR. ${ }^{18-20}$

As COVID-19 is a new disease emerging in late 2019, there are no longterm follow-up patient data available for reference. To approximate the effect of COVID-19 on OS after TAVR, we used available data on the impact of in-hospital infections, the principle entity of which is pneumonia, after TAVR on 2-year OS based on hazard ratios. ${ }^{30,31}$ The decrease in 2-year OS for prompt TAVR due to COVID-19 was determined to be $9 \%$ to $16 \%$ for intermediate risk and $2.6 \%$ to $4.5 \%$ for low risk. The decrease in 2 -year OS for urgent or emergent TAVR due to COVID-19 was set to be the same for both intermediate-risk and low-risk cases (14\% to $26 \%$ ) given the same change in status.

\section{Sensitivity Analyses}

Numerous variables contribute to an individual patients' risk of sudden death while awaiting TAVR, risk of disease progression requiring urgent TAVR, and 2-year OS after having undergone TAVR. Because of the variability and uncertainty in estimating these values, we performed multiple one-way sensitivity analyses to account for the uncertainty in these key model parameters and to approximate how differences in the base case could affect the model outcome. This is accomplished by altering one parameter at a time while holding all other variables constant at baseline values. One-way sensitivity analyses were performed for both low-risk and intermediate-risk patients by varying the probabilities of requiring urgent or emergent TAVR during the delay, of dying due to disease progression during the delay, the 2-year OS of TAVR, the probability of perioperative COVID-19 infection, and the probability of mortality from COVID-19. Much uncertainty remains today with regard to the risk of acquiring COVID-19 and the patient-specific risk of dying from

TABLE 2. Model parameters

\begin{tabular}{|c|c|c|c|c|c|}
\hline \multirow[b]{2}{*}{ Chance parameter } & \multicolumn{2}{|c|}{ Intermediate risk } & \multicolumn{2}{|c|}{ Low risk } & \multirow[b]{2}{*}{ Reference(s) } \\
\hline & $\overline{\text { Probability }}$ & Range & $\overline{\text { Probability }}$ & Range & \\
\hline $\begin{array}{l}\text { TAVR operative mortality, } \\
\text { elective }\end{array}$ & 0.0399 & - & 0.015 & - & ACC/STS calculator \\
\hline $\begin{array}{l}\text { TAVR operative mortality, } \\
\text { urgent }\end{array}$ & 0.0612 & - & 0.023 & - & $18,19,20$ \\
\hline COVID-19 mortality & 0.21 & $0.1-0.35$ & 0.21 & $0.1-0.35$ & 21,22 \\
\hline $\begin{array}{l}\text { Prompt TAVR } \\
\text { Perioperative COVID-19 } \\
\text { infection }\end{array}$ & 0.014 & $0.01-0.20$ & 0.014 & $0.01-0.20$ & $*$ \\
\hline $\begin{array}{l}\text { Delayed TAVR } \\
\text { Perioperative COVID-19 } \\
\quad \text { infection }\end{array}$ & 0.00001 & $0-0.01$ & 0.00001 & $0-0.01$ & $*$ \\
\hline Elective TAVR & 0.59 & - & 0.70 & & Remainder from urgent/death \\
\hline Urgent/emergent TAVR & 0.26 & $0.162-0.357$ & 0.26 & $0.162-0.357$ & $5,23,24$ \\
\hline $\begin{array}{l}\text { No surgery (disease } \\
\text { progression) }\end{array}$ & 0.15 & $0.045-0.237$ & 0.05 & 0-0.09 & $5,6,23,25,26$ \\
\hline
\end{tabular}

$\overline{T A V R}$, Transcatheter aortic valve replacement; ACC, American College of Cardiology; STS, Society of Thoracic Surgeons; COVID-19, 2019 novel coronavirus. *Parameters set by the research team based on current local data on asymptomatic transmission rates. 
TABLE 3. Two-year OS

\begin{tabular}{|c|c|c|c|c|c|}
\hline \multirow[b]{2}{*}{ Timing of TAVR } & \multicolumn{2}{|c|}{ Intermediate risk } & \multicolumn{2}{|c|}{ Low risk } & \multirow[b]{2}{*}{ Reference(s) } \\
\hline & Value & Range & Value & Range & \\
\hline \multicolumn{6}{|l|}{ Without COVID-19 infection } \\
\hline Prompt TAVR & 0.85 & $0.83-.087$ & 0.965 & $0.955-0.976$ & 29 \\
\hline Urgent/emergent TAVR & 0.76 & $0.74-0.79$ & 0.76 & $0.74-0.79$ & $18,19,20$ \\
\hline \multicolumn{6}{|l|}{ With COVID-19 infection } \\
\hline Prompt TAVR & 0.72 & $0.69-0.74$ & 0.93 & $0.91-0.95$ & 30,31 \\
\hline Urgent/emergent TAVR & 0.56 & $0.497-0.623$ & 0.56 & $0.497-0.623$ & 30,31 \\
\hline
\end{tabular}

The 2-year OS data for low-risk and intermediate-risk patients are based on COVID-19 infection status and urgency of TAVR. Data are extrapolated using hazard ratios for hospital-acquired pneumonia following TAVR. TAVR, Transcatheter aortic valve replacement; COVID-19, 2019 novel coronavirus.

COVID-19. To model the uncertainty in these COVID-19 variables, 2-way sensitivity analysis was performed (Tables 2 and 3 ) by simultaneously varying the probability of acquiring perioperative COVID-19 and the probability of mortality from COVID-19 once acquired for both low- and intermediate-risk patients while holding all other parameters constant. To model the effect of a longer or shorter length of delay, an additional 2 -way sensitivity analysis was performed by simultaneously varying the probability of undergoing urgent or emergent TAVR and the probability of dying before undergoing TAVR for both low-risk and intermediaterisk patients.

\section{RESULTS}

Proceeding with prompt TAVR for intermediate-risk AS resulted in greatly improved 2-year OS compared with delaying TAVR for 6 months to allow for COVID-19 prevalence to decrease (2-year OS, 0.81 for prompt TAVR and 0.67 for delayed TAVR). The 2-year OS for the low-risk patient proceeding with prompt TAVR was also improved compared with delaying TAVR for 6 months (2-year OS, 0.95 for prompt TAVR and 0.85 for delayed TAVR). These results were essentially unchanged when the prevalence of COVID-19 at 6 months remained at $0.7 \%$.

\section{Sensitivity Analyses: Intermediate Risk}

Altering the probability of requiring urgent or emergent TAVR or dying before undergoing TAVR did not change the outcome of favoring prompt TAVR. Prompt TAVR was favored for a COVID-19 infection probability of $<55 \%$, and delayed TAVR was favored for a COVID-19 infection probability of $>55 \%$.

Results of the 2-way sensitivity analysis altering the probability of acquiring COVID-19 and the probability of mortality from an acquired COVID-19 infection are shown in Figure 2. This figure demonstrates that prompt TAVR is favored for a wide range of probabilities both of acquiring COVID-19 and of dying from COVID-19. Even if the probability of dying from an acquired COVID-19 infection is $>50 \%$, the probability of acquiring such infection would have to be $>40 \%$ for delayed TAVR to be the favored strategy (Figure 2). If the probability of undergoing urgent TAVR is $\leq 3 \%$ and the probability of dying before undergoing TAVR is $\leq 1 \%$, then delaying the procedure is favored (Figure E1); however, this scenario is clinically unlikely, given the evidence suggesting that delaying TAVR by even 3 months results in at least a $16 \%$ risk of requiring urgent TAVR during that interval (Table 2).

\section{Sensitivity Analyses: Low Risk}

Altering the probability of requiring urgent or emergent TAVR or dying before undergoing TAVR did not change the outcome of favoring prompt TAVR. Prompt TAVR was favored for a COVID-19 infection probability $<42 \%$, whereas delayed TAVR was favored for a COVID-19 infection probability $>47 \%$.

Two-way sensitivity analysis again demonstrates that for a wide range of probabilities of acquiring COVID-19 and mortality from COVID-19, prompt TAVR is favored. If the probability of COVID-19 mortality is $>50 \%$, then the probability of perioperative COVID-19 infection must be $>30 \%$ for delayed TAVR to be favored (Figure 3). If the probability of undergoing urgent TAVR is $\leq 3 \%$ and the

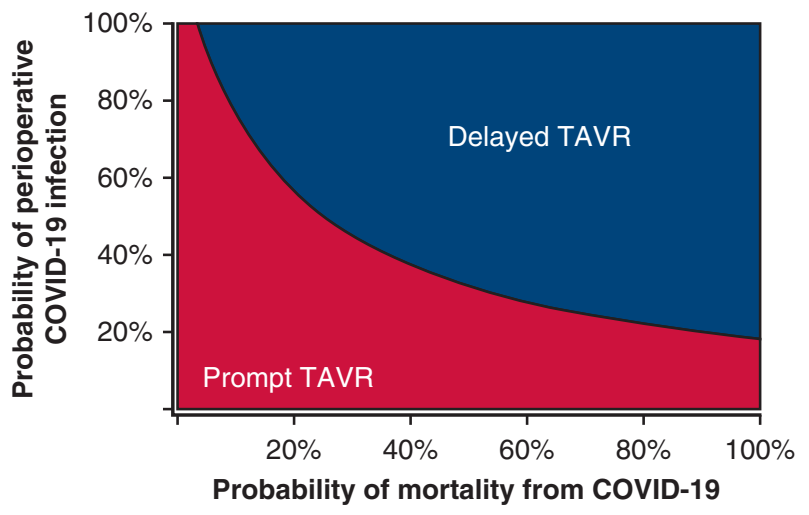

FIGURE 2. Two-way sensitivity analysis for the probabilities of infection and mortality from coronavirus disease 2019 (COVID-19) in intermediaterisk patients. The graph displays the favored strategy (prompt or delayed transcatheter aortic valve replacement $[T A V R]$ ) across a range of all possible probabilities of contracting perioperative COVID-19 infection and COVID-19-related mortality while holding all other model variables constant at baseline values. For any given probability of mortality from COVID-19, one can estimate the probability of contracting COVID-19 in which the favored strategy changes from prompt TAVR to delayed TAVR. The vast majority of patients will benefit from prompt TAVR because they will fall into the left lower quadrant of the figure with a probability of either contracting COVID-19 or dying from it of $<40 \%$. 
probability of dying before undergoing TAVR is $<0.5 \%$, then delaying the procedure is favored (Figure E2). Again, this scenario is clinically unlikely, as the evidence suggests that delaying TAVR for even 3 months results in at least a $16 \%$ risk of requiring urgent TAVR during that interval (Table 2).

\section{DISCUSSION}

COVID-19 is likely to continue to tax healthcare resources, and a resurgence is a highly likely possibility. This decision analysis will help guide clinicians and healthcare systems in deciding how to triage TAVR patients based on the local prevalence of COVID-19. Because long-term survival with AS can be significantly impacted by delays in diagnosis and treatment, ${ }^{5,6}$ we created an informative simple model quantifying the potential harm to a patient with severe symptomatic AS if TAVR was delayed due to the COVID-19 pandemic, the results of which are summarized in Figure 4. Multiple consensus statements and expert opinions have been published with regard to this clinical question without any data-driven analyses. In a recently published case series, 77 patients with severe AS were followed as their procedure was delayed secondary to COVID$19{ }^{24}$ During their 3-month follow-up period while elective TAVR was delayed, 3 patients $(4 \%)$ died while awaiting TAVR, and 24 patients $(31 \%)$ underwent urgent TAVR for accelerated symptoms; both values are consistent with the ranges which we used for our modeling.

Our decision analysis model found that delaying TAVR for 6 months impacted the 2-year OS in patients that were either intermediate risk ( 0.81 for prompt TAVR vs 0.67 for delayed TAVR) or low risk ( 0.95 for prompt TAVR vs 0.85 for delayed TAVR). In addition, when we performed a 2-way sensitivity analysis to vary probabilities of disease progression, including probabilities that were representative of a shorter delay, prompt TAVR was still preferred over delayed TAVR. Furthermore, the model suggests a significant risk of acquiring perioperative COVID-19 (>47\%) is required to favor delaying TAVR, at which point most hospitals would have insufficient resources to proceed with TAVR regardless. To date, no US community has seen this degree of community prevalence during the pandemic. Examining the communities with the highest burden of COVID-19 disease necessitating the complete shutdown of operative and procedural services reveals a prevalence threshold much lower than that required in our simulation for delayed TAVR to be preferred. This implies that as long as the healthcare system in question has resources to offer TAVR, they should be doing so.

Although decision analysis model parameters are very narrow in scope by design, the sensitivity analyses allow for modeling a range of uncertainty. The 2-way sensitivity analysis may serve as a helpful guide for clinicians to interpret the model for different patient- and system-level factors

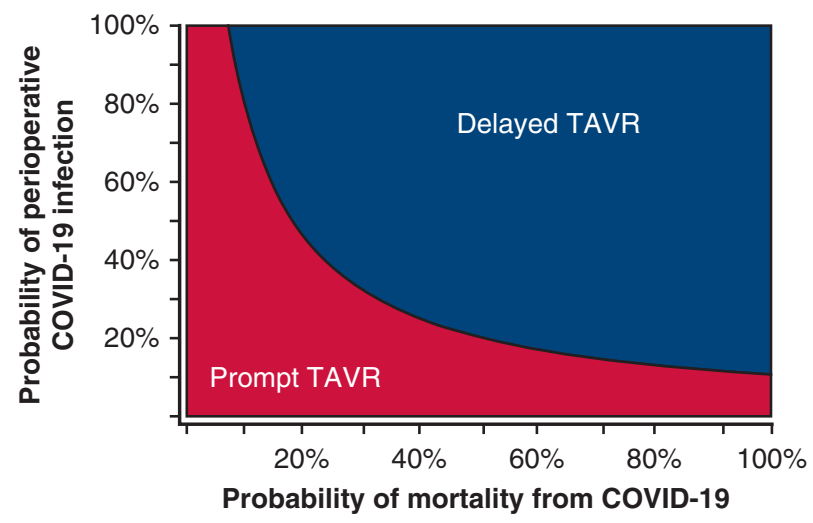

FIGURE 3. Two-way sensitivity analysis for the probability of infection and mortality from coronavirus disease 2019 (COVID-19) in low-risk patients. The graph displays the favored strategy (prompt or delayed transcatheter aortic valve replacement $[T A V R]$ ) across a range of possible probabilities of contracting perioperative COVID-19 infection and COVID-19-related mortality while holding all other model variables constant at baseline values. For any given probability of mortality from COVID-19, one can estimate the probability of contracting COVID-19 in which the favored strategy changes from prompt TAVR to delayed TAVR. The vast majority of patients will benefit from prompt TAVR because they will fall into the left lower quadrant of the figure with a probability of either contracting COVID-19 or dying from it of $<40 \%$.

at the time of intervention. For example, older patients with multiple comorbidities who are at intermediate risk for TAVR are more likely to have severe infections and increased mortality from COVID-19. Using Figure 2, if mortality exceeds $60 \%$ for these patients, then delayed resection is likely preferred when infection prevalence exceeds $25 \%$. Conversely, among younger patients with few comorbidities and a lower mortality rate of $10 \%$, the decision to delay resection may be preferred only when infection prevalence exceeds $80 \%$. It is unlikely that any patient will fall into the predicted mortality range for a given incidence of COVID-19, which means there are very few, if any, patient-specific factors that should lead one to delay TAVR.

Although this analysis was not designed to evaluate the decision making process for patients undergoing surgical AVR, it is interesting to note the similarities in the data between the 2 groups. Two-year OS and in-hospital mortality are not statistically significantly different between the 2 procedures; therefore, the only parameter likely to differ between the 2 groups is the risk of acquiring COVID-19 in the postoperative period, likely to be higher in the surgical group owing to a higher percentage of patients requiring in-patient rehabilitation or an additional caregiver at home while recovering. This is accounted for in the 2-way sensitivity analysis described in Figures 2 and 3, which would seem to suggest that prompt AVR is preferred for almost all patients. 

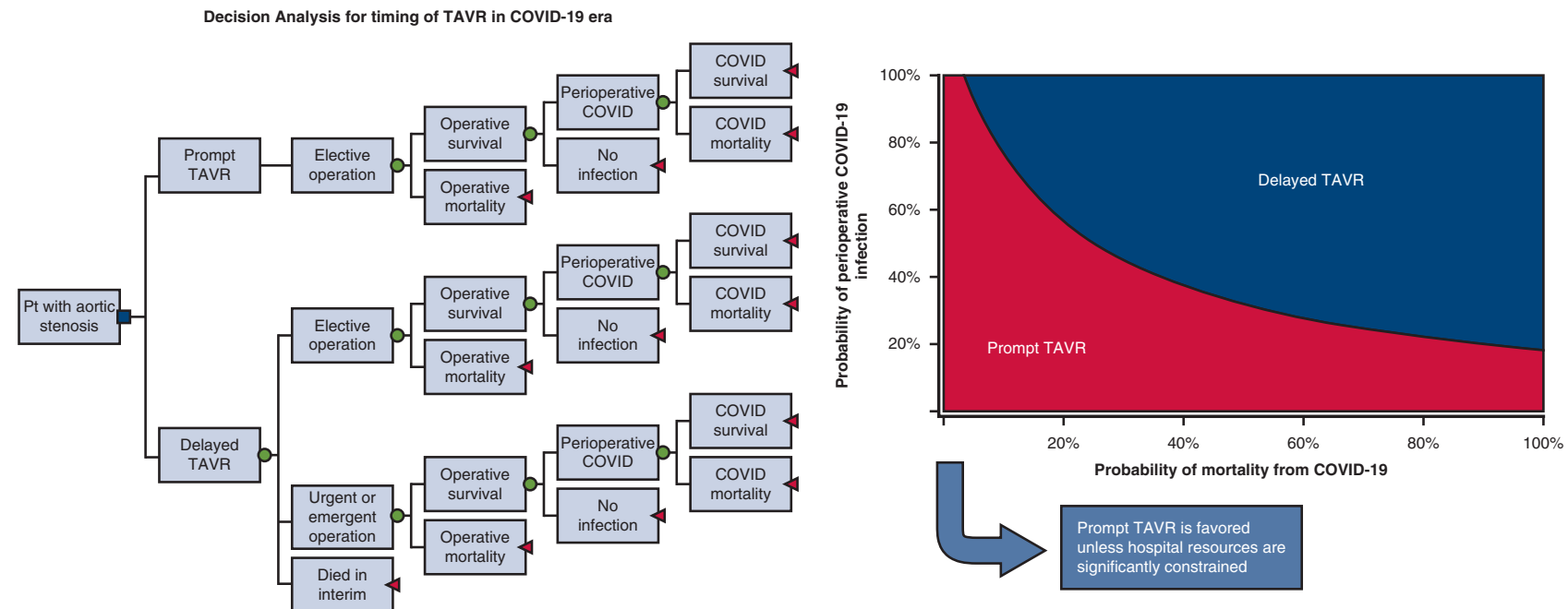

TAVR-transcatheter aortic valve replacement, COVID-19- novel coronavirus 2019

FIGURE 4. Decision analysis for timing of transcatheter aortic valve replacement (TAVR) in the coronavirus disease 2019 (COVID-19) era. The decision analysis tree demonstrates possible outcomes for patients with severe aortic stenosis. The model predicts the benefit of prompt TAVR versus delayed TAVR based on the probabilities of acquisition or mortality from COVID-19 and favors prompt TAVR for the majority of probabilities likely to be encountered (red region).

One limitation of this study is the paucity of available literature on COVID-19, which resulted in estimating several model parameters from similar but nonidentical clinical scenarios. We addressed this by analyzing a range of values for the COVID-19 parameters in our sensitivity analyses. Second, defining the perioperative risk of acquiring COVID-19 is difficult, as it is becoming increasingly clear that individual behaviors and exposures weigh heavily on one's risk of exposure. We addressed this by using the asymptomatic prevalence as our risk because it is a good marker of the risk of coming into contact with someone in the community who unknowingly has the disease, and also because this number is readily available from local health departments. In addition, predicting the mortality rate for a patient who develops perioperative COVID-19 after undergoing TAVR is handicapped by incomplete available evidence. We reference 2 case series showing a postoperative mortality of $20 \%$ following a heterogenous group of surgeries in a total of 75 patients.

Finally, another limitation of our study is our inability to predict the risk of COVID-19 transmission in 6 months. As the pandemic has progressed, it has become increasingly clear that a variety of social and political factors weigh heavily on the curve of transmission for a given community. Our model assumes the institution of several well-described public health mitigation efforts, such as mask wearing and social distancing, which have demonstrated an ability to reduce transmission rates and local community prevalence. It has become increasingly clear that the vast majority of COVID-19 transmissions are occurring in the community and not within the hospital; therefore, it is a reasonable assumption that delaying in-hospital procedures does not significantly reduce the risk of acquiring COVID-19.
Furthermore, as our 2-way sensitivity analysis demonstrates, an individual's risk of mortality from COVID-19 would have to be extremely high to warrant delaying TAVR, even with a high community prevalence.

Resource utilization is an important element of medical decision making during a pandemic. In the present analysis, we assumed that the treating center had adequate resources to proceed with TAVR. As we have seen during the COVID19 pandemic, some healthcare systems have become overwhelmed, and limitations in ICU beds, ventilators, personal protective equipment, blood product supply, and clinical staff which may preclude TAVR. Institutions across the country have developed alternative staffing models to deal with surges in critically ill coronavirus patients. These models often include physicians and nursing staff being reallocated from one service arena to another. Which areas are negatively affected by staff being pulled will vary from institution to institution and can potentially impact the ability to fully staff operating rooms, cardiac catheterization labs, ICUs, or postprocedural step-down units. All these factors must be taken into consideration when determining whether a particular institution is sufficiently resourceconstrained that they cannot adequately care for TAVR patients. However, as the prevalence of COVID-19 fluctuates in communities, or other infectious pandemics arise, this model can be adapted to help hospitals and surgeons determine when to proceed with specific operations.

\section{CONCLUSIONS}

Proceeding with prompt TAVR for low- and intermediate-risk patients with symptomatic severe AS during the COVID-19 pandemic resulted in an improved 2-year OS compared with delaying TAVR for a period as short as 3 
to 6 months. This improved survival was more pronounced for patients at intermediate risk but held true even for lowrisk patients. As the risk of perioperative COVID-19 increases above $55 \%$ (intermediate risk) or $47 \%$ (low risk), delaying TAVR is associated with improved long-term survival; however, with these rates of COVID-19 infection, local hospitals would be overwhelmed and would have insufficient resources to proceed with TAVR. According to our analysis, the only reason to delay TAVR would be insufficient resources to care for these patients in the perioperative period.

\section{Conflict of Interest Statement}

The authors reported no conflicts of interest.

The Journal policy requires editors and reviewers to disclose conflicts of interest and to decline handling or reviewing manuscripts for which they may have a conflict of interest. The editors and reviewers of this article have no conflicts of interest.

\section{References}

1. World Health Organization. Coronavirus disease (COVID-19) events as they happen. Available at: https://www.who.int/emergencies/diseases/novel-corona virus-2019/events-as-they-happen. Accessed April 6, 2020.

2. Grasselli G, Zangrillo A, Zanella A, Antonelli M, Cabrini L, Castelli A, et al. Baseline characteristics and outcomes of 1591 patients infected with SARSCoV-2 admitted to ICUs of the Lombardy region, Italy. JAMA. 2020;323: 1574-81.

3. American College of Surgeons. COVID-19: recommendations for management of elective surgical procedures. Available at: https://www.facs.org/covid-19/ clinical-guidance/elective-surgery. Accessed May 2, 2020.

4. Centers for Medicare and Medicaid Services. Non-emergent, elective medical services, and treatment recommendations. Available at: https://www.cms. gov/files/document/cms-non-emergent-elective-medical-recommendations.pdf. Accessed May 2, 2020.

5. Shimura T, Yamamoto M, Kano S, Hosoba S, Sago M, Kagase A, et al. Patients refusing transcatheter aortic valve replacement even once have poorer clinical outcomes. J Am Heart Assoc. 2018;7:e009195.

6. Malaisrie SC, McDonald E, Kruse J, Li Z, McGee EC Jr, Abicht TO, et al. Mortality while waiting for aortic valve replacement. Ann Thorac Surg. 2014;98: 1564-71.

7. Shah PB, FGP Welt, Mahmud E, Phillips A, Kleiman N, Young MN, et al; American College of Cardiology Society for Cardiovascular Angiography and Interventions. Triage considerations for patients referred for structural heart disease intervention during the COVID-19 pandemic: an ACC/SCAI position statement. JACC Cardiovasc Interv. 2020;13:1484-8.

8. Mehra MR, Desai SS, Kuy S, Henry TD, Patel AN. Cardiovascular disease, drug therapy, and mortality in Covid-19. N Engl J Med. 2020;382:e102.

9. Arentz M, Yim E, Klaff L, Lokhandwala S, Riedo FX, Chong M, et al. Characteristics and outcomes of 21 critically ill patients with COVID-19 in Washington State. JAMA. 2020;323:1612-4.

10. Sterpetti AV. Lessons learned during the COVID-19 virus pandemic. J Am Coll Surg. 2020;230:1092-3.

11. Wu C, Chen X, Cai Y, Xia J, Zhou X, Xu S, et al. Risk factors associated with acute respiratory distress syndrome and death in patients with coronavirus disease 2019 pneumonia in Wuhan, China. JAMA Intern Med. 2020;180:934-43.

12. Onder G, Rezza G, Brusaferro S. Case-fatality rate and characteristics of patients dying in relation to COVID-19 in Italy. JAMA. 2020;323:1775-6.

13. Verity R, Okell LC, Dorigatti I, Winskill P, Whittaker C, Imai N, et al. Estimates of the severity of coronavirus disease 2019: a model-based analysis [published correction appears in Lancet Infect Dis. 2020 Apr 15]. Lancet Infect Dis. 2020;20:669-77.

14. Korean Society of Infectious Diseases, Korean Society of Pediatric Infectious Diseases, Korean Society of Epidemiology, Korean Society for Antimicrobial
Therapy, Korean Society for Healthcare-associated Infection Control and Prevention, Korea Centers for Disease Control and Prevention. Report on the epide miological features of coronavirus disease 2019 (COVID-19) outbreak in the Republic of Korea from January 19 to March 2, 2020. J Korean Med Sci. 2020;35:e112.

15. Richardson S, Hirsch JS, Narasimhan M, Crawford JM, McGinn T, Davidson KW, et al. Presenting characteristics, comorbidities, and outcomes among 5700 patients hospitalized with COVID-19 in the New York City Area. JAMA. 2020;323:2052-9.

16. Kumar A, Sato K, Narayanswami J, Banerjee K, Andress K, Lokhande C, et al. Current Society of Thoracic Surgeons model reclassifies mortality risk in patients undergoing transcatheter aortic valve replacement. Circ Cardiovasc Interv. 2018; 11:e006664.

17. Okoh AK, Fugar S, Kang N, Haik N, Choi M, Cohen M, et al. Predictors of extended postoperative length of stay after uncomplicated transcatheter aortic valve replacement. J Invasive Cardiol. 2019;31:153-8.

18. Elbadawi A, Elgendy IY, Mentias A, Saad M, Mohamed AH, Choudhry MW, et al. Outcomes of urgent versus nonurgent transcatheter aortic valve replacement. Catheter Cardiovasc Interv. 2020;96:189-95.

19. Ichibori Y, Li J, Patel T, Lipinski J, Ladas T, Saric P, et al. Short-term and long-term outcomes of patients undergoing urgent transcatheter aortic valve replacement under a minimalist strategy. J Invasive Cardiol. 2019; 31:E30-6.

20. Kolte D, Khera S, Vemulapalli S, Dai D, Heo S, Goldsweig AM, et al. Outcomes following urgent/emergent transcatheter aortic valve replacement: insights from the STS/ACC TVT registry. JACC Cardiovasc Interv. 2018;11: $1175-85$.

21. Lei S, Jiang F, Su W, Chen C, Chen J, Mei W, et al. Clinical characteristics and outcomes of patients undergoing surgeries during the incubation period of COVID-19 infection. EClinicalMedicine. 2020;21:100331.

22. Doglietto F, Vezzoli M, Gheza F, Lussardi GL, Domenicucci M, Vecchiarelli L, et al. Factors associated with surgical mortality and complications among patients with and without coronavirus disease 2019 (COVID-19) in Italy. JAMA Surg. 2020;155:691-702.

23. Bouma B, van den Brink RB, van der Meulen JHP, Verheul H, Cheriex E, Hamer H, et al. To operate or not on elderly patients with aortic stenosis: the decision and its consequences. Heart. 1999;82:143-8.

24. Ro R, Khera S, Tang GHL, Krishnamoorthy P, Sharma SK, Kini A, et al. Characteristics and outcomes of patients deferred for transcatheter aortic valve replacement because of COVID-19. JAMA Netw Open. 2020;3:e2019801.

25. Oh JK, Park JH, Hwang JK, Lee CH, Park JS, Park JI, et al. Long-term survival in Korean elderly patients with symptomatic severe aortic stenosis who refused aortic valve replacement. Korean Circ J. 2019;49:160-9.

26. Elbaz-Greener G, Masih S, Fang J, Ko DT, Lauck SB, Webb JG, et al. Temporal trends and clinical consequences of wait times for transcatheter aortic valve replacement: a population-based study. Circulation. 2018;138: 483-93.

27. American College of Cardiology, Society of Thoracic Surgeons. TAVR inhospital mortality risk calculator. Available at: https://tools.acc.org/tavrrisk/ \#!/content/evaluate/. Accessed April 29, 2020.

28. Society of Thoracic Surgeons. STS risk calculator. Available at: http://riskcalc sts.org/stswebriskcalc/calculate. Accessed April 29, 2020.

29. Leon MB, Smith CR, Mack MJ, Makkar RR, Svensson LG, Kodali SK, et al. Transcatheter or surgical aortic-valve replacement in intermediate-risk patients. N Engl J Med. 2016;374:1609-20.

30. Tirado-Conte G, Freitas-Ferraz AB, Nombela-Franco L, Jimenez-Quevedo P, Biagioni C, Cuadrado A, et al. Incidence, causes, and impact of in-hospital infections after transcatheter aortic valve implantation. Am J Cardiol. 2016;118: 403-9.

31. Nombela-Franco L, del Trigo M, Morrison-Polo G, Veiga G, Jimenez-Quevedo P, Altisent OA, et al. Incidence, causes, and predictors of early ( $\leq 30$ days) and late unplanned hospital readmissions after transcatheter aortic valve replacement. JACC Cardiovasc Interv. 2015;8:1748-57.

32. Reardon MJ, Van Mieghem NM, Popma JJ, Kleiman NS, Søndergaard L, Mumtaz M, et al. Surgical or transcatheter aortic-valve replacement in intermediate-risk patients. $N$ Engl J Med. 2017:376:1321-31.

Key Words: aortic stenosis, COVID-19, transcatheter aortic valve replacement 


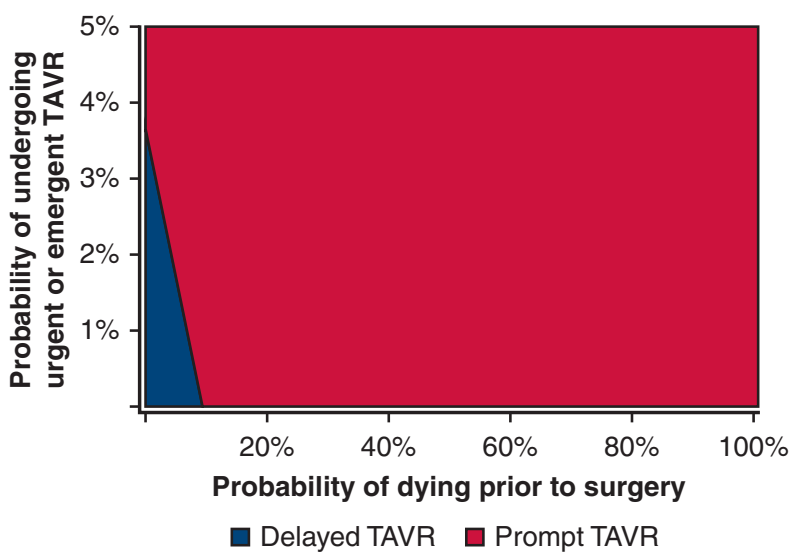

FIGURE E1. Two-way sensitivity analysis for probability of undergoing urgent or emergent transcatheter aortic valve replacement (TAVR) or dying before repair during a 6-month delay due to the coronavirus disease 2019 (COVID-19) pandemic in intermediate-risk patients. The graph displays the favored strategy (prompt or delayed TAVR) across a range of possible probabilities of dying before surgery or requiring urgent/emergent repair while holding all other model variables constant at baseline values. Prompt TAVR is preferred in all clinically relevant circumstances, as the probability of undergoing urgent/emergent TAVR within 3 to 6 months is $>5 \%$ based on the best available evidence, easily falling in the prompt TAVR area of $>5 \%$.

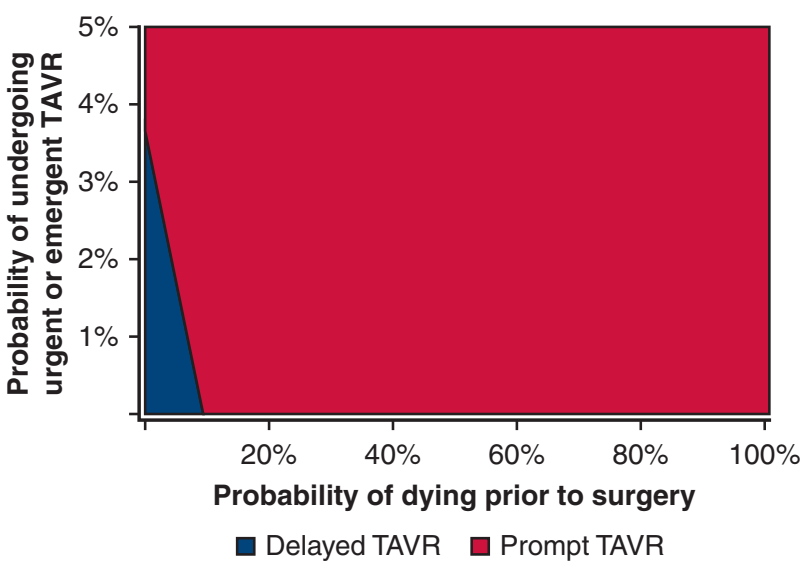

FIGURE E2. Two-way sensitivity analysis for the probability of undergoing urgent or emergent transcatheter aortic valve replacement (TAVR) or dying before repair during the 6-month delay due to the coronavirus disease 2019 (COVID-19) pandemic in low-risk patients. The graph displays the favored strategy (prompt or delayed TAVR) across a range of possible probabilities for dying before surgery or requiring urgent/emergent repair while holding all other model variables constant at baseline values. Prompt TAVR is preferred in all clinically relevant circumstances, as the probability of undergoing urgent/emergent TAVR within 3 to 6 months is $>5 \%$ based on the best available evidence, easily falling in the prompt TAVR area of $>5 \%$. 